\title{
Criminologie
}

\section{Politique institutionnelle de libération : identification des individus dangereux. Une revue de la littérature}

\section{Vernon L. Quinsey}

Volume 17, numéro 2, 1984

La dangerosité, un débat à poursuivre

URI : https://id.erudit.org/iderudit/017199ar

DOI : https://doi.org/10.7202/017199ar

Aller au sommaire du numéro

\section{Éditeur(s)}

Les Presses de l'Université de Montréal

ISSN

0316-0041 (imprimé)

1492-1367 (numérique)

Découvrir la revue

Citer ce document

Quinsey, V. L. (1984). Politique institutionnelle de libération : identification des individus dangereux. Une revue de la littérature. Criminologie, 17(2), 53-78.

https://doi.org/10.7202/017199ar
Résumé de l'article

Follow-up studies of the physical dangerousness of men released from correctional institutions, mental hospitals and special security psychiatric institutions have indicated that we cannot as yet predict with an acceptable degree of accuracy which men will be dangerous upon release. Because of the methodological and conceptual weaknesses of these predictive studies and their lack of success, as well as the practical difficulties which would arise from not attempting to select nondangerous men for release, a new approach to the problem of prediction is required. Such an approach might profitably make predictions according to how different sorts of offenders respond to various situations. Theories taking into account the interactions between persons and their environment may in future allow tolerably accurate predictions of future assaultive behavior under certain circumscribed conditions. 


\section{POLITIQUE INSTITUTIONNELLE DE LIBÉRATION IDENTIFICATION DES INDIVIDUS DANGEREUX UNE REVUE DE LA LITTÉRATURE* Vernon L. Quinsey**}

Follow-up studies of the physical dangerousness of men released from correctional institutions, mental hospitals and special security psychiatric institutions have indicated that we cannot as yet predict with an acceptable degree of accuracy which men will be dangerous upon release. Because of the methodological and conceptual weaknesses of these predictive studies and their lack of success, as well as the practical difficulties which would arise from not attempting to select nondangerous men for release, a new approach to the problem of prediction is required. Such an approach might profitably make predictions according to how different sorts of offenders respond to various situations. Theories taking into account the interactions between persons and their environment may in future allow tolerably accurate predictions of future assaultive behavior under certain circumscribed conditions.

\section{INTRODUCTION}

La littérature, portant sur la prédiction du comportement violent des individus libérés d'institutions psychiatriques ou correctionnelles, est abondante et controversée. Le développement d'une méthode fiable permettant de prédire la dangerosité des personnes libérées aurait des conséquences profondes sur les politiques institutionnelles. Cependant, et nous en discuterons plus loin, il existe un consensus important, voire l'unanimité, à l'effet que les évaluations de la dangerosité sont très conservatrices et de peu de validité. Étant donné ce consensus, il est étonnant de constater que ces évaluations continuent à être faites sur un grand nombre d'individus (pour une excellente discussion historique de ce sujet, voir Petrunik, 1982). Cette situation est due, en partie au fait que, même si ces évaluations de la dangerosité des individus se révèlent peu valides, il s'avère extrêmement difficile de s'en passer en milieu institutionnel, pour des raisons d'ordre pratique; et qui plus est, les exclure ne contribuerait sans doute pas à élargir les politiques de libération.

* Je remercie les docteurs G. Harris et M. Rice de leurs commentaires sur une version précédente de ce manuscrit. Ce texte reprend, en partie, un article écrit grâce au financement du ministère fédéral du Solliciteur général (Quinsey, Ambtman, et Pruesse, 1977). Il a été traduit de l'anglais par Fernande Rossini et Claudette Lavallée.

** Dr. Vernon Quinsey, chercheur invité, service de la recherche, Institut Philippe Pinel de Montréal. 
Puisqu'il est probable qu'on continuera à procéder à des évaluations de la dangerosité dans les milieux institutionnels en dépit de leur peu de validité, il s'avère important de faire le point sur ce que nous en savons et de voir comment nous pourrions améliorer nos méthodes de prédiction. Nous nous proposons de restreindre, autant que possible, cette revue de la littérature à l'examen d'études de relance sur la dangerosité d'individus libérés de diverses institutions.

\section{ÉTUDES DE RELANCE}

\section{INDIVIDUS LIBÉRÉS D'INSTITUTIONS CORRECTIONNELLES}

Les plus vastes études de relance sur la dangerosité de délinquants libérés sont sans doute celles menées par la California Youth Authority (Molof, 1965; Wenk, Robinson et Smith, 1972). Ces études portant sur de jeunes délinquants ont démontré que les tentatives de prédire lesquels parmi eux commettraient des délits avec violence après leur libération résultaient en un nombre inacceptable d'erreurs de classification d'individus présumés dangereux. Le problème à la base de ces tentatives de prédiction était la rareté des crimes avec violence effectivement commis par les individus relâchés. Étant donné le nombre restreint de récidives à caractère violent, la prédiction la plus exacte consisterait à n'en prévoir aucune.

Walker, Hammond et Steer (1967) ont suivi, durant onze ans, les dossiers judiciaires de 4301 Écossais qui avaient été déclarés coupables d'une infraction en 1947. Ces chercheurs ont également obtenu les casiers judiciaires antérieurs de 4239 Londoniens déclarés coupables d'un délit au cours de 1957 ainsi que leurs dossiers couvrant les cinq années subséquentes. Les infractions de "violence contre la personne" comprenaient le meurtre, les menaces, l'utilisation d'armes à feu, les vols qualifiés et les assauts, mais n'incluaient pas la cruauté à l'égard des enfants, la négligence criminelle par suite de conduite dangereuse ou les agressions sexuelles avec violence. Parmi les Écossais, 264 individus ont commis des délits avec violence en 1947 ou plus tard, et parmi les Londoniens, 500 ont récidivé utilisant la violence. Dans les deux échantillons, la probabilité qu'un individu soit de nouveau reconnu coupable de délit de violence augmentait légèrement avec chaque infraction non violente. Cependant, avec chaque condamnation successive pour délit de violence, la probabilité de récidive augmentait beaucoup plus nettement. Dans l'échantillon des Écossais, par exemple, $14 \%$ des 264 individus qui avaient été déclarés coupables d'un délit de violence l'ont aussi été d'un autre délit, alors que $40 \%$ des 45 personnes avec deux condamnations antérieures pour violence ont été de nouveau condamnés pour des 
délits de violence; finalement, $55 \%$ des 11 individus avec quatre condamnations ou plus pour délits de violence ont été condamnés encore pour des crimes impliquant la violence. Ces données révèlent qu'un passé de violence répétée pourrait être utilisé comme outil de prédiction pour cette minorité d'individus qui ont, plusieurs fois déjà, été condamnés pour des délits de violence.

Nuffield (1982) a fait le suivi pendant trois ans, de 2475 individus libérés des pénitenciers canadiens. Étaient considérés comme récidive de violence, l'homicide, l'assaut, l'agression sexuelle grave, le vol à main armée et le vol qualifié. Une proportion de $12,6 \%$ des individus ont été arrêtés de nouveau pour crimes violents (presque la moitié des délits impliquaient des vols à main armée ou qualifiés). La méthode de prédiction de Burgess (qui consiste à assigner un poids à chacune des variables ayant une valeur discriminante et à faire la somme de ces poids pour chaque délinquant) a divisé la moitié de l'échantillon utilisé à des fins de validation, en quatre catégories de risque dont le taux de réussite (i.e. aucun délit de violence) a été de $69,82,87$ et $93 \%$. Malheureusement, le degré de précision de cette méthode de prédiction, quoique supérieur à celui obtenu à l'aide des méthodes de régression ou de prédiction par attributs dichotomiques, n'a pas été suffisamment élevé pour que la méthode soit utilisée à des fins de prédiction. Il semble cependant très clair, à partir de ces données, que les détenus dont on peut être sûr qu'ils ne récidiveront pas de façon violente peuvent être identifiés avec certitude. Les variables dont le poids était le plus élevé dans la méthode de prédiction de la violence de Burgess étaient : le jeune âge lors de la première condamnation; des condamnations antérieures pour délits de violence; des condamnations antérieures pour assauts non sexuels; des antécédents d'évasions; cinq incarcérations antérieures ou plus; le délit en cause actuellement (délit avec violence, agression sexuelle ou possession illégale d'arme à feu) et le jeune âge à l'admission.

\section{INDIVIDUS LIBÉRÉS D'HÔPITAUX SÉCURITAIRES}

Un second champ d'études de relance se rapporte aux patients libérés d'institutions psychiatriques à sécurité maximum. Sont gardés dans ces institutions des patients trouvés atteints d'aliénation mentale pendant leur incarcération, inaptes à subir leur procès, déclarés non coupables pour aliénation mentale et/ou qui présentent des problèmes graves de contrôle de leur dangerosité dans les hôpitaux psychiatriques.

Steadman et Cocozza (1974) ont colligé des données sur la dangerosité de 967 patients transférés des hôpitaux pour criminels aliénés de l'État de New York, suite à la décision Baxstrom. Ces chercheurs ont 
constaté que seulement 24 de ces patients ont été réadmis dans des hôpitaux sécuritaires. Soulignons cependant que ces individus étaient plus âgés que les sujets observés dans la plupart des autres études de relance. Leur moyenne d'âge lors du transfert était de 47 ans. Thornberry et Jacoby (1979) ont obtenu des résultats semblables dans le follow-up de patients libérés en Pennsylvanie. Un petit nombre seulement des patients de cet échantillon se sont révélés dangereux après leur libération. Toutefois, comme dans les études effectuées après la décision Baxstrom, les patients étaient relativement âgés et il semble qu'ils n'aient pas été détenus parce que les cliniciens en médecine légale les avaient déclarés dangereux à la suite d'observations rigoureuses.

Kozol et ses collègues (Kozol, Boucher et Garofalo, 1972; Kozol, 1975) ont préconisé une approche clinique à la prédiction de la violence lors de la libération. Ces chercheurs ont publié une liste de principes directeurs à consulter avant de poser un diagnostic, un cadre de référence pour faciliter l'évaluation de la dangerosité. Ce cadre de référence prend la forme de questions, que l'examinateur doit se poser au sujet de la personne évaluée, telles que : «Est-ce que la vie a un sens pour lui?», "A-t-il manifesté de la cruauté de façon agressive et injustifiée?", «S'est-il identifié d'une façon ou d'une autre à sa victime?». Kozol et al. ont utilisé ces principes directeurs pour ensuite faire part des résultats de cette recherche menée auprès de 561 criminels masculins du Massachussets renvoyés en observation. La majorité des individus avaient commis des délits sexuels où la victime n'était pas consentante. Le personnel a diagnostiqué 304 de ces 561 individus comme non dangereux; ils ont été libérés après avoir purgé leur peine. 226 autres ont été déclarés dangereux et internés pour un traitement d'une durée indéterminée. De ces derniers, 83 ont été libérés par le personnel de l'institution une fois le traitement psychothérapeutique terminé et 49 patients ont été remis en liberté par les tribunaux à l'encontre de la recommandation du personnel clinique. Le taux de récidive pour assaut grave après leur libération a été de $9 \%$ pour les individus déclarés non dangereux, $6 \%$ pour ceux qui ont été libérés par ordre de la cour. Kozol et al. ont interprété ces résultats comme une validation de leur méthode clinique d'évaluation. Il est, cependant, très difficile d'accepter ces résultats tels quels car il n'y est pas précisé si les índividus libérés par la cour et ceux libérés ou déclarés non dangereux par l'institution ont vécu dans la collectivité durant des périodes de temps comparables.

Quatre études de relance ont été menées auprès de patients libérés de l'institution psychiatrique à sécurité maximum d'Oak Ridge en Ontario (Quinsey, 1979). Dans la première de ces études, 91 patients qui avaient 
été remis en liberté par le Comité de révision, après que leur requête auprès des autorités de l'hôpital eut été rejetée, ont fait l'objet d'un suivi. Seize de ces 91 patients ont commis un acte de violence contre la personne (incluant menaces et vol à main armée mais excluant possession d'arme) dans l'intervalle de la première à la quatrième année suivant leur libération. Sur quinze variables à l'étude, une seule différenciait de façon significative les patients violents des non violents : les patients violents libérés avaient commis plus fréquemment des crimes contre la personne avant leur internement à cette institution. Dans une étude subséquente, 56 patients déclarés inaptes à subir leur procès ou non coupables pour cause d'aliénation ont fait l'objet d'un suivi pendant une période moyenne de 30 mois après leur libération. Un seul des patients a été reconnu coupable d'assaut. Dans une troisième étude, un groupe de 60 patients libérés d'un hôpital sécuritaire a fait l'objet d'un suivi pendant une durée moyenne de 39 mois. Ces patients furent répartis en trois groupes égaux : des patients déclarés inaptes à subir leur procès ou non coupables pour cause d'aliénation, des patients internés contre leur gré et libérés par le Comité de révision à l'encontre de l'avis de l'hôpital et finalement, les patients libérés par l'hôpital. Les deux premiers groupes furent sélectionnés à partir de l'échantillon utilisé pour les études précédentes. Même si on a trouvé des variables qui différenciaient les patients libérés reconnus coupables d'un nouveau délit ou réadmis à l'hôpital, le nombre d'individus ayant commis des actes de violence contre la personne était trop restreint pour permettre des analyses statistiques. Dans la dernière étude de cette série, tous les patients traités puis libérés ou transférés d'Oak Ridge vers d'autres institutions en $1972(\mathrm{~N}=206)$ ont fait l'objet d'un suivi pendant une période de trois à quatre ans. Trente-quatre patients (17\%) ont été condamnés ou renvoyés à Oak Ridge à cause d'un délit de violence contre la personne. Les patients souffrant de troubles de la personnalité de même que les plus jeunes avaient davantage tendance à être violents après leur libération. Le délit le plus fréquent était l'assaut simple. Peu de ces délits ont effectivement causé des blessures corporelles, bien qu'un individu ait commis un homicide dans une prison et qu 'un autre ait été accusé de tentative de meurtre lors d'un incident dans une école d'apprentissage. Il va de soi qu'un certain nombre de "malades mentaux-criminels" sont dangereux, toutefois, cette recherche s'accorde avec d'autres études de relance pour suggérer que peu de patients relâchés des hôpitaux sécuritaires sont dangereux pour autrui.

Cependant, toutes les études de relance abordent la prédiction de la dangerosité indirectement, puisqu'on peut présumer que tous les individus détenus étaient, en réalité, extrêmement dangereux. Les études portant sur des groupes d'individus libérés en même temps (Baxstrom et 
Dixon) présentent des problèmes à cet égard, parce qu'il semble que les patients aient été détenus à cause de l'inertie et de l'incompétence bureaucratique plutôt qu'à la suite d'une évaluation rigoureuse. Dans le but de contourner ces difficultés d'interprétation, nous avons examiné directement la justesse de la prise de décision des cliniciens à la fois dans des situations réelles et dans des conditions expérimentales. La logique sous-jacente à ces études pourrait s'exprimer ainsi : si les cliniciens ne sont pas d'accord entre eux, ils ne peuvent pas tous avoir raison; en d'autres mots, le degré de désaccord limite la validité de la prédiction.

La première étude portait sur des patients déclarés non coupables pour cause d'aliénation ou (plus rarement) sur des patients jugés inaptes à subir leur procès; elle a été conçue dans le but d'évaluer le degré de corrélation entre les évaluations des cliniciens (Quinsey et Ambtman, 1978). À ce sujet, trois psychiatres et un psychologue ont complété un questionnaire pendant ou après une conférence tenue pour chaque étude de cas dans le but de décider si le patient était éligible à une libération. Les évaluations de la dangerosité rendues par les quatre cliniciens étaient, comme prévu, fortement reliées aux recommandations de chacune des études de cas, mais le taux de corrélation moyen entre les cliniciens sur ces évaluations était de 0,60 , indiquant un accord modéré. Cependant, le fait que les cliniciens aient discuté du cas avant de procéder à son évaluation a sans doute entraîné une surestimation de l'accord entre les évaluateurs.

La seconde étude de cette série (Quinsey et Ambtman, 1979) a porté sur une situation expérimentale d'évaluation plutôt que sur une situation réelle dans le but d'examiner : a) la corrélation entre les évaluations des cliniciens sans l'avantage de discussions préalables et b) l'apport de différents types d'information à l'évaluation de la dangerosité du patient. Trente patients ont été sélectionnés en fonction des critères suivants : le crime pour lequel ils ont été admis était-il un crime de violence non sexuelle contre un adulte (habituellement un meurtre), un délit sexuel grave sur un enfant de 13 ans ou moins, ou un délit contre la propriété? Trois types d'informations furent recueillies pour chacun des patients : la description du délit, l'histoire antérieure et l'évaluation clinique (incluant les tests psychologiques, l'état mental et la progression du traitement). Ces informations se sont chevauchées seulement au niveau de l'âge du patient et du nombre de mois de l'hospitalisation en cours qui se retrouvaient dans les trois sources. Les données étaient évaluées deux fois par chacun des évaluateurs; en une première occasion, chaque type d'information leur a été donné séparément, comme s'il concernait différents patients et, la deuxième fois, les trois sortes d'informations leur ont été 
présentées ensemble comme s'il s'agissait d'un seul dossier (ce qui était effectivement le cas). Les deux séances d'évaluation avaient lieu à cinq semaines d'intervalle au moins et l'ordre de la présentation variait pour chacun des évaluateurs. Quatre psychiatres et neuf enseignants du niveau secondaire ont, chacun de leur côté, évalué les trois types d'information séparément puis ensemble, quant à la probabilité que le patient commette un délit de violence, qu'il se rende coupable d'un délit contre la propriété, et quant à la gravité du délit de violence advenant le cas où il serait commis. Toutes les opinions étaient émises comme si le patient devait être libéré au moment de l'évaluation; de plus, les évaluateurs devaient indiquer si oui ou non le patient devrait être effectivement relâché.

Les taux de corrélation entre les différentes évaluations faites par chacun des experts, enseignants ou psychiatres, ont été assez bas sur les trois types d'informations, qu'ils aient été présentés séparément ou ensemble, peu importe ce qu'ils avaient à évaluer. Même si les taux de corrélation entre les différentes évaluations d'un même expert étaient bas, les taux entre les moyennes des deux groupes occupationnels avaient tendance à être plutôt élevés. Par ailleurs, les psychiatres et les enseignants ne s'entendaient pas entre eux quant au choix des patients qui devraient être libérés. On a découvert que la description du délit était le seul type d'informations ayant une influence sur l'évaluation du dossier complet quant à la dimension «gravité du délit de violence advenant le cas où il serait commis".

Ces deux études indiquant que des conférences cliniques ne peuvent mener à des prédictions valides de la dangerosité d'un patient, que les informations produites pour ces évaluations cliniques n'ont guère de valeur dans le processus d'évaluation lui-même et que les jugements des psychiatres sont fort probablement semblables à ceux qu'un profane instruit pourrait faire.

\section{PROBLÈMES MÉTHODOLOGIQUES}

Comme nous l'avons mentionné dans la section précédente, la valeur des prédictions de la dangerosité est minime en dépit du fait que la science a beaucoup progressé depuis dix ou vingt ans dans la compréhension du comportement agressif antisocial. Dans le but de développer des stratégies pour la recherche future, il s'avère important d'établir pourquoi il existe si peu d'information utile à la prédiction dans ce domaine. Avant de suggérer quelques solutions au problème, nous effectuerons une revue des problèmes méthodologiques communs à un grand nombre d'études de relance. 


\section{LE PROBLÈME DU TAUX DE BASE}

Comme nous l'avons vu, plusieurs des problèmes de prédiction de la dangerosité proviennent de la rareté du comportement qu'on cherche à prédire. En effet, des crimes de violence, tels que le meurtre, sont rarement commis par des individus relâchés d'institutions psychiatriques. Comme l'ont signalé plusieurs auteurs, les tentatives visant à prédire des événements rares conduisent inévitablement à de nombreuses affirmations erronées (individus classés dangereux par erreur).

Un taux de base (ou taux d'occurrence) peu élevé de violence après la libération peut avoir un impact important sur la validité des décisions de remettre ou non un individu en liberté. Pour illustrer ces effets, supposons que $100(10 \%)$ individus sur 1000 commettraient un délit grave contre la personne s'ils étaient tous libérés. Si nous pouvions développer un instrument de prédiction d'une validité exceptionnelle permettant de différencier les individus effectivement dangereux des sujets non dangereux avec une précision de $80 \%$ pour chacun des deux groupes, et d'appliquer ensuite cet instrument pour décider lesquels parmi les 1000 individus nous devrions relâcher, nous ferions 200 erreurs $(20 \%)$. Ces 200 erreurs seraient de deux types : il y aurait 20 individus dangereux libérés comme non dangereux (i.e. $20 \%$ des 100 individus vraiment dangereux) et 180 sujets non dangereux classés par erreur comme dangereux ( $20 \%$ des 900 individus non dangereux). $\mathrm{Si}$, d'autre part, tout le groupe était libéré sans évaluation préalable, nous ferions 100 erreurs, la moitié de ce que nous ferions avec une méthode de prédiction valide à $80 \%$. Notons que toutes les erreurs faites en libérant simplement tous les sujets sont des erreurs dues à la témérité et elles affectent toute la communauté alors qu'en utilisant la méthode de prédiction, 180 des 200 erreurs sont dues à la prudence et affectent surtout les individus concernés (Quinsey, 1980). Les conséquences d'un taux d'occurrence trop bas ont été directement responsables de l'échec de l'approche sentencielle de neutralisation sélective visant à contrer la récidive avec violence (Greenwood et Abrahamse, 1982). Dans cette thèse, on utilise les crimes révélés par les détenus eux-mêmes pour évaluer la fréquence des délits; auront de plus longues sentences les détenus ayant les mêmes caractéristiques que ceux qui ont commis de nombreux délits. En ce qui a trait au contrôle de la violence, cependant, Greenwood et Abrahamse concluent : "... des études antérieures ont montré qu'il est extrêmement difficile de prédire ces délits ou d'en réduire le nombre par la neutralisation sélective parce que le taux d'occurrence auquel ils sont commis par un quelconque délinquant est trop faible." 
Malheureusement, et l'efficacité des décisions cliniques en souffre, des études psychologique de prédiction ont démontré que les gens, qui essaient de prédire des événements à partir de sources d'information diverses, ne tiennent généralement aucun compte des taux d'occurrence de ces événements même quand ils sont conscients du peu de validité de ces prédicteurs (Kahneman et Tversky, 1973).

Le dilemme auquel fait face une institution, dont les procédures de libération sont trop imprécises, est qu'elle n'a aucun moyen, mis à part la libération d'individus choisis au hasard, d'évaluer la proportion de détenus réellement dangereux. Il est évident que les individus libérés par l'institution ne peuvent être considérés comme ayant été choisis au hasard. Quand la proportion d'individus dangereux n'est pas connue, il est difficile de choisir un taux de sélection qui soit rationnel, d'évaluer l'efficacité de la prise de décision, ou même de savoir s'il est avantageux d'essayer de développer des techniques d'évaluation plus efficaces. On pourrait contourner partiellement ce problème en choisissant des critères d'élargissement plus libéraux jusqu'à ce que le nombre d'individus commettant des délits avec violence après leur libération devienne inacceptable. Cette solution ne semble cependant pas politiquement réalisable; de plus, elle suppose que les dimensions significatives sont connues. Le fait que des individus soient détenus pour des sentences maximum de durée déterminée est, d'une certaine manière, préférable puisque chacun d'eux peut être suivi et le taux de base déterminé pour la population incarcérée. Malheureusement, plus les sentences deviennent longues, plus la difficulté de définir le taux de base se rapproche de celle qu'on rencontre dans le cas des sentences indéterminées; par exemple, même si tous les individus purgeant des sentences déterminées de 20 ans sont relâchés éventuellement, on ne peut présumer qu'ils sont représentatifs des sujets libérés après avoir purgé 5 ans seulement de leur sentence de 20 ans.

En résumé, nous pouvons conclure que, non seulement les taux d'occurrence peu élevés de comportements dangereux mènent à une surprédiction de la dangerosité mais, il est extrêmement difficile d'évaluer le taux de base d'un grand nombre de populations internées. $L$ 'incapacité d'établir ce taux au sein de la population empêche les intervenants de progresser dans l'élaboration de politiques rationnelles de libération.

Aussi insoluble qu'il puisse paraître, le problème du taux de base n'est pas universel. Monahan (1978) souligne qu'on peut retrouver des taux de base élevés dans les cas d'internement d'urgence d'aliénés mentaux et qu'alors, on peut s'attendre à une plus grande précision au niveau de la prédiction parce que le contexte de la prédiction et le 
comportement à prédire sont associés à la fois au niveau temporel et situationnel, en somme, dans ce cas, la situation est souvent inchangée. Les comparutions pour cautionnements en sont un autre exemple.

Au sujet des décisions de libération prises par l'institution, Quinsey (1980) a démontré d'une façon détaillée que le taux de base des individus réellement dangereux peut augmenter de façon dramatique au sein d'une institution si une cohorte d'individus est évaluée à plusieurs reprises en vue d'un élargissement, à partir de critères peu efficaces. Ces réflexions renforcent notre conviction qu'il est rentable de vouloir développer des mesures de prédiction efficaces à utiliser dans certains contextes particuliers.

\section{OMISSION DES VARIABLES SITUATIONNELLES}

La plupart des prédictions au sujet du comportement dangereux sont élaborées uniquement à partir d'informations sur le délit commis par l'individu, sur son histoire et sur ses caractéristiques personnelles (Steadman, 1976). Il n'y a pas eu de tentatives spécifiques pour tenter d'identifier les stimuli ayant provoqué les délits de violence antérieurs, pour établir si ces éléments seraient ou ne seraient pas présents dans le milieu de vie de cet individu après sa libération et pour en tenir compte dans une équation de prédiction. Étant donné qu'un comportement violent est souvent provoqué par la présence de certains stimuli, il est inconcevable de tenter de prédire l'occurrence d'une réponse à certains types de stimuli sans savoir lesquels provoqueraient une réaction sur un individu donné et à quelle fréquence ils se produisent. Quoique plusieurs auteurs aient souligné cette difficulté, peu de progrès a été réalisé en ce sens jusqu'à tout récemment. Steadman (1982), par exemple, a étudié la violence de malades mentaux libérés, de délinquants relâchés et de la population en général, dans un contexte situationnel. Monahan et Klassen (1982) ont fait la revue du peu de connaissances acquises à propos des déterminants situationnels de la violence.

\section{DONNÉES BASÉES SUR DES CRITĖRES INADÉQUATS}

Les données les plus fréquemment utilisées en prédiction sont les rapports de police ayant servi aux condamnations à la cour criminelle. Les criminologues se sont souvent plaints des nombreux problèmes rencontrés dans l'interprétation des informations contenues dans les dossiers de la police. Ce que les études de relance essaient de mesurer, c'est le taux d'occurrence du comportement dangereux mais, ce qu'elles mesurent en réalité, ce sont les comportements dangereux pour lesquels les sujets de leur échantillon se sont faits prendre la première fois pour 
être ensuite inculpés et condamnés, en supposant que les négociations lors du plaidoyer n'ont pas changé la nature du délit en un autre impliquant plutôt un comportement non dangereux. Ainsi, nous ne mesurons pas tant le comportement dangereux du délinquant que sa chance, l'efficacité de la police, l'opinion sociale face au délit et la dénonciation du processus judiciaire de type accusatoire.

Une autre difficulté provient du fait que les auteurs utilisent souvent pour leur recherche des définitions différentes du comportement dangereux. Des infractions criminelles telles que le vol qualifié ou le port d'armes illégal sont tantôt considérées dangereuses, tantôt non dangereuses. Le fait que la définition soit plus ou moins large a un effet considérable sur la capacité des variables à discriminer les individus libérés dangereux et les non dangereux et sur le taux d'occurrence du phénomène. Les chercheurs diffèrent également en ceci que certains choisissent l'arrestation, d'autres la condamnation, d'autres encore des sources différentes d'information comme critère du comportement. De telles différences méthodologiques rendent les études réplicatives difficile à effectuer et empêchent les généralisations empiriques.

Un dernier problème réside dans la prédiction de la récidive avec violence prise globalement car la récidive avec violence regroupe des infractions n'ayant sur le plan théorique aucun rapport entre elles; elles peuvent aller du meurtre avec agression sexuelle jusqu'au vol à main armée. Ce regroupement peut augmenter le taux d'occurrence mais, les composantes n'étant pas reliées, le résultat restera imprévisible.

\section{DONNÉES INADÉQUATES POUR LA PRÉDICTION}

Les études qui ont utilisé des opinions cliniques comme outil de prédiction présentent souvent des listes de critères employés lors des évaluations de la dangerosité mais aucune explication sur la façon dont ces critères ont été mis en relation pour en arriver à une décision. De plus, on ne fournit aucune information au sujet de la concordance entre les jugements des différents évaluateurs, basés sur ces critères. Quoique l'évaluation clinique soit un processus difficile à décrire, l'incapacité d'en fournir une description objective rend toute reproduction de ce processus impossible.

D'autres études, qui ont utilisé des tests psychologiques, donnent souvent l'impression qu'on a choisi de tels tests simplement parce qu'ils étaient disponibles dans les dossiers et non parce qu'ils avaient été conçus dans le but de classer les individus libérés comme dangereux ou non dangereux. Ainsi, les tests MMPI et Rorschach ont été les plus utilisés. II 
est encourageant toutefois de constater que plusieurs tentatives ont été faites pour modifier d'anciens tests ou en développer de nouveaux permettant de prédire le comportement violent.

Les données historiques telles l’âge de l'individu, le sexe, l'état matrimonial, les condamnations antérieures ont peut-être une importance théorique mais semblent avoir été sélectionnées pour des raisons d'ordre pratique. Le fait que certaines de ces variables semblent effectivement en mesure d'augmenter la capacité de prédiction est en lui-même un hommage au pouvoir des méthodes empiriques brutes face à certains problèmes. Le fait que ces variables d'accès facile n'aient pas été analysées avec beaucoup d'imagination présente une faiblesse supplémentaire à leur utilisation. Il est possible que des analyses séquentielles de ces variables, qui tiennent compte de l'ordre et du moment de certaines expériences vécues, auraient plus de force et de sens, d'un point de vue théorique, que l'utilisation de prédicteurs statiques (Wilkins, 1973).

\section{VOIES POSSIBLES POUR LA RECHERCHE FUTURE}

Actuellement, il ne paraît pas y avoir de méthode satisfaisante de prédiction de la dangerosité future des individus en institution. À partir de là, deux conclusions sont possibles. La première est que le taux d'occurrence peu élevé de la violence et la nature des déterminants situationnels de cette dernière sont insolubles avec les techniques actuelles et que les efforts pour tenter de prédire la violence devraient être abandonnés ou, au mieux, axés vers une libéralisation et une plus grande justice dans les politiques de libération. Une meilleure conclusion s'impose cependant. En effet, la recherche n'a pas été orientée jusqu'à présent, autant qu'elle aurait pu l'être, vers la découverte d'une méthode de prédiction efficace. Comme l'a démontré cette revue de la littérature, les études de relance ne sont pas nombreuses et sont émaillées d'obstacles méthodologiques dont quelques-uns sont certes surmontables. De toute façon, on continuera à faire des prédictions dans un avenir prévisible. La seule alternative raisonnable, par conséquent, semble être d'utiliser nos connaissances pour améliorer les prédictions cliniques et pour perfectionner notre méthodologie de recherche en misant sur les points forts et en tirant une leçon des points faibles des études déjà effectuées.

Monahan (1981) a bien résumé comment les prédictions cliniques, dans l'état actuel des choses, devaient être effectuées. Il n'offre pas d'algorithme mais plutôt une série de points que la documentation de recherche propose comme importants. Brièvement, ces points englobent : a) les événements qui ont provoqué la violence antérieure ou les menaces et leur contexte spécifique; b) les caractéristiques démographi- 
ques pertinentes de l'individu; c) l'histoire de violence antérieure du sujet; d) le taux d'occurrence de la violence parmi les individus présentant les mêmes caractéristiques; e) les tensions actuelles; f) les facteurs cognitifs et affectifs dénotant une prédisposition à réagir violemment ou non violemment au stress; g) les similarités du futur milieu de vie avec le milieu antérieur qui a provoqué la violence; h) la disponibilité future de victimes éventuelles et i) la disponibilité de moyens permettant de commettre des actes de violence. Cette liste de points qu'un clinicien doit prendre en considération, quoique la meilleure que la littérature ait à offrir, montre qu'il y a encore de grands efforts à fournir avant qu'il soit possible de faire des prédictions exactes. Il faudra procéder à des recherches supplémentaires avant de pouvoir suggérer des solutions concrètes aux cliniciens.

\section{VARIABLES DE PRÉDICTION POSSIBLES}

Il existe une vaste littérature sur la relation entre les variables individuelles et le comportement agressif même quand on exclut les études de laboratoire sur la violence. Malheureusement, la plupart de ces études ont établi un lien entre les variables individuelles et le comportement agressif à posteriori; en d'autres mots, on a tenté de trouver des variables permettant de différencier les individus qui ont été violents dans le passé de ceux qui ne l'ont pas été. La valeur prédictive des variables identifiées dans les études effectuées à posteriori est discutable, mais il semble que ce serait une stratégie raisonnable que de valider quelquesunes des variables les plus prometteuses relevées dans les études prédictives plutôt que de les choisir parce qu'elles sont facilement accessibles. Parce qu un grand nombre d'articles ont été publiés sur ce sujet et qu'il existe plusieurs excellentes revues de la littérature (ex. Megargee, 1970; 1982; Monahan, 1981), nous ne tenterons pas de revoir de façon exhaustive la littérature ayant trait aux études de prédiction à posteriori.

\section{VARIABLES DÉMOGRAPHIQUES ET HISTORIQUES}

Il a été démontré dans des études de prédiction qu'un grand nombre de variables démographiques et historiques n'ont qu'une faible relation avec la dangerosité postérieure à la libération. Par exemple, on sait que, même si les délinquants violents atteints de troubles mentaux ont moins tendance à récidiver que les délinquants non violents atteints de troubles mentaux, ils ont davantage tendance à récidiver en commettant un nouveau délit de violence (Quinsey, Wareford, Pruesse et Link, 1975; Tong et McKay, 1959). Un certain nombre d'études (ex. Molof, 1965) ont montré que l'âge présente un lien avec la récidive de violence subséquente parmi les jeunes détenus. 
Comme nous l'avons montré cependant, l'utilisation de telles informations démographiques et historiques courantes ne nous a pas permis de faire de prédictions exactes dans le passé. Ce genre de données peut néanmoins être très utile dans la prédiction en permettant de limiter les catégories d'individus au sujet desquels nous avons fait des prédictions. Par exemple, pour nous permettre d'augmenter le taux de base du phénomène à l'étude, nous pourrions exclure les individus de plus de 40 ans et ceux qui n’ont pas commis antérieurement de délit de violence.

\section{VARIABLES PSYCHIATRIQUES}

Le rapport du groupe de travail de l'American Psychiatric Association portant sur les aspects cliniques de l'étude des individus violents (1974) a conclu que les prédictions cliniques de la dangerosité sont fondamentalement peu fiables.

Plusieurs études ont examiné la fréquence des désordres psychiatriques parmi les populations de criminels dans le but de déterminer quelle est la relation entre le diagnostic psychiatrique et le délit de violence. Malheureusement, les résultats de ces études sont peu concluants parce que les différents chercheurs ont utilisé des procédés de diagnostic et des indices de pathologie différents (Mesnikoff et Lanterbach, 1975). Le diagnostic décrit le plus fréquemment comme étant relié à la dangerosité est la psychopathie. Il y a des indices (Pruesse et Quinsey, 1977) témoignant d'une relation entre un diagnostic de désordre de la personnalité et les délits de violence après la libération, mais l'étude de relance la plus vaste effectuée sur des psychopathes démontre que cette relation n'est pas assez étroite pour être utilisée dans la prédiction de cas individuels. Dans cette étude, Gibbens, Pond et Stafford-Clark (1959) ont suivi, pendant une période de 8 ans, 72 psychopathes «graves" incarcérés. Les psychopathes étaient sélectionnés d'après certaines variables telles que le manque de réaction à la punition, la révolte contre les parents, un mauvais dossier d'emploi et l'absence de projets ou de buts. Vingt-quatre \% de cet échantillon n'avait pas été inculpé de nouveau même si tous avaient été en liberté pendant au moins quatre ans. Selon les rapports de police, un total de 16 détenus ont commis au moins un délit de violence après leur libération; cependant, la majorité de ces délits étaient mineurs. Il y a eu trois délits graves : un détenu a été accusé d'homicide involontaire et deux autres d'agression grave contre la personne. Ces données démontrent que les délinquants psychopathes "graves" ne sont pas aussi dangereux qu'on le pense couramment.

Dans une étude auprès de 5525 patients, Hedlund, Altman et Evenson (1973) ont tenté de prédire à posteriori quels patients avaient été 
dangereux envers les autres en utilisant un grand nombre d'items informatisés sur l'état mental ainsi que des variables démographiques, concernant l'admission ou liées au diagnostic. Le taux de faux positifs produit par l'équation de régression variait de $60 \%$ pour la prédiction à posteriori du comportement violent à $80 \%$ pour le fait d'avoir réellement blessé quelqu'un. Manifestement, le taux d'occurrence peu élevé de violence physique parmi cette population psychiatrique excluait la possibilité d'une prédiction clinique à posteriori qui soit exacte. Des études de patients libérés d'hôpitaux psychiatriques démontrent avec régularité que les patients atteints de maladie mentale ne sont pas particulièrement dangereux (Gulevich et Bourne, 1970).

En accord avec d'autres revues de la littérature (ex. Mesnikoff et Lanterbach, 1975), il serait juste de conclure que la relation n'est pas forte entre le diagnostic psychiatrique ou l'évaluation pathologique et la dangerosité. Des variables psychiatriques telles le diagnostic seraient utilisées à meilleur escient conjointement avec d'autres variables, par exemple pour prédire quel type de victimes seraient «choisies" par divers groupes d'individus identifiés comme présentant de fortes probabilités de manifester un comportement violent dans le futur (ainsi par exemple, cette comparaison pourrait révéler des différences entre les victimes de récidivistes psychopathes et celles des récidivistes paranoïdes). Il y aurait lieu de penser à mener des études permettant de découvrir si l'usage de psychotropes réduit la probabilité de comportements violents parmi des patients libérés ou parmi les détenus présentant des désordres mentaux et sélectionnés selon certains critères psychiatriques.

\section{VARIABLES PSYCHOMÉTRIQUES}

On a étudié la relation entre de nombreux tests psychologiques projectifs ou non projectifs et le comportement agressif. Toutefois, la plupart de ces études ont utilisé des mesures de l'agressivité utilisant papier et crayon ou se sont servies des tests permettant de distinguer les individus violents des non violents à posteriori. Très peu d'études ont tenté de prédire quels individus commettraient des délits de violence dans l'avenir. Megargee $(1970 ; 1982)$ a fait l'inventaire de la littérature sur la prédiction du comportement violent à partir de tests psychologiques et en a conclu qu'aucun test n'est reconnu actuellement comme étant valable en prédiction.

Il existe cependant quelques tests psychologiques qui ont permis de différencier les délinquants violents des non violents à posteriori. Par exemple, Megargee, Cook et Mendelsohn (1967) ont mis au point une échelle à partir des items du MMPI qui a permis de différencier les 
délinquants agressifs surcontrôlés des sous-contrôlés. Leur recherche indique l'intérêt qu'il y a à développer des instruments pour mesurer des caractéristiques spécifiques à certaines catégories particulières de délits. Ils ont d'abord démontré que leur instrument de mesure permettait de distinguer les prisonniers extrêmement agressifs (condamnés pour meurtre ou agression avec une arme offensive), à la fois des détenus modérément agressifs (condamnés pour voies de fait) et de ceux qui ne le sont pas. À partir de ces résultats et du contenu des items utilisés, ils ont déduit que leur échelle mesure jusqu’à quel point un individu surcontrôle son hostilité et ils ont élaboré la théorie suivante : quand les inhibitions massives d'un individu agressif qui surcontrôle sa violence sont vaincues par une provocation extrême, une réaction extrêmement violente se produit. La personne surcontrôlée serait toutefois, dans la plupart des cas, peu sûre d'elle, sursocialisée et non criminelle. Plusieurs études viennent appuyer cette hypothèse. Une première (Megargee, Cook et Mendelsohn, 1967) permet de constater que l'échelle de l'hostilité surcontrôlée $(\mathrm{OH}=$ overcontrolled hostility) différenciait les détenus très agressifs qui avaient été jugés surcontrôlés de ceux jugés sous-contrôlés; les opinions étaient formulées à partir d'un résumé d’histoire de cas incluant des informations tel le nombre de délits antérieurs.

D'autres études ont tenté d'établir un rapport entre les scores à l'échelle $\mathrm{OH}$ et des corrélations théoriquement significatives. White, McAdoo et Megargee (1973) ont constaté que les détenus ayant un score OH élevé avaient, tel que prédit, plus de maturité, plus de maîtrise de soi et étaient plus consciencieux; ils avaient par ailleurs moins d'assurance, moins de tendances paranoïdes; ils étaient moins sophistiqués et plus conventionnels que les détenus ayant un taux $\mathrm{OH}$ plus bas. Quinsey, Maquire et Varney (1983) ont démontré que les meurtriers au score $\mathrm{OH}$ élevé avaient moins d'assurance (tant au niveau des mesures comportementales qu'autocomplétées) que les meurtriers présentant un score $\mathrm{OH}$ peu élevé et les autres patients d'un hôpital psychiatrique à sécurité maximum. Ces résultats ont été confirmés par Henderson (1983) qui a constaté que les délinquants au score $\mathrm{OH}$ élevé formulaient moins d'interventions négatives que les autres délinquants et par Walters et Greene (1983) qui ont découvert un facteur de conformité sociale dans l'échelle d'hostilité surcontrôlée $(\mathrm{OH})$. Étant donné que l'utilité de l'échelle $\mathrm{OH}$ a été démontrée dans les études de prédiction à posteriori, il est malheureux qu'elle n'ait pas été plus largement utilisée dans les études de relance.

\section{TYPOLOGIES D'INDIVIDUS VIOLENTS}

Une façon évidente d'augmenter notre habileté à prédire les délits de violence serait de classer les délinquants dans un certain nombre de types 
ou catégories pour lesquels les taux de récidive, les stratégies d'intervention et les variables prédictives varieraient. Un tel travail a déjà été fait dans plusieurs secteurs de la criminologie. Par exemple, une typologie d'individus qui se sont livrês à des voies de fait sur les enfants, basée sur le type de victimes, a été associée à la récidive, bien qu'aucune typologie n'ait été, à ce jour, fortement associée à la dangerosité de ces individus (Quinsey, 1977). Classer les violeurs en fonction de certains types s`annonce un travail prometteur mais n'a pas encore été l'objet de tests rigoureux (Quinsey, sous presse). Il y a des preuves cependant que chez les violeurs, l'excitation sexuelle provoquée par la violence non sexuelle telle que mesurée par la turgescence pénienne est associée au degré de souffrance physique infligée aux victimes précédentes (Quinsey et Chaplin, 1982).

Plusieurs typologies, qui semblent valables, ont été suggérées pour les meurtriers (Megargee, 1982); elles ne sont pas ratifiées dans le détail par des preuves empiriques mais elles sont compatibles avec l'information disponible. Conrad (1966), par exemple, a proposé sept types de délinquants violents dont les plus importants sont : le violent par culture qui provient d'une sous-culture où la violence était acceptée; le criminel violent qui utilise la violence pour des gains pécuniaires; le violent pathologique qui est un malade mental; et le violent situationnel qui commet un acte d'agression dans un cas de provocation extrême.

\section{VARIABLES NEUROPHYSIOLOGIQUES}

Il n'y a aucune mesure physiologique reconnue, à ce jour, comme devant être utile dans la prédiction de la dangerosité. La revue de la littérature portant sur le lien entre le cerveau et la violence, publiée dans les Archives of Neurology (Goldstein, 1974) fait autorité; elle conclut : "Bien qu'il y ait une littérature considérable sur le meurtre, il appert que sa prédiction est difficile et que le rôle joué par des facteurs "organiques" tels les tumeurs au cerveau, la démence, l'épilepsie, etc., est minime mais mérite des recherches plus approfondies." Personne, cependant, ayant lu quelques-unes des impressionnantes études neurophysiologiques sur l'agression effectuée sur des animaux (ex. Flynn, 1967), ne peut douter de l'avenir prometteur de la recherche neurophysiologique. Nous avons besoin d'études de contrôle menées sur une grande échelle et portant sur les réactions physiologiques de délinquants violents à des stimuli et à des situations soigneusement choisis. Une telle approche expérimentale a donné de bons résultats dans d'autres domaines comme dans la mesure des préférences sexuelles des individus ayant molesté des enfants, à partir de l'enregistrement de la réaction pénienne 
lors de projections de diapositives de personnes d'âges et de sexes variés (Quinsey, 1977). Mednick, Pollock, Voayka et Gabrielli (1982) ont examiné la littérature sur les approches physiologiques et ont décrit les résultats prometteurs d'études portant sur l'influence respective de l'hérédité et du milieu et ceux d'études utilisant les mesures physiologiques dans un contexte longitudinal.

\section{7. ÉLABORATION D'UNE THÉORIE DE LA VIOLENCE}

Une grande part du travail effectué dans le domaine de la prédiction de la violence a négligé l'aspect théorique du problème et a semblé suivre la voie du moindre effort. La majorité des études ont utilisé des mesures de prédiction et se sont basées sur certains critères plutôt que d'autres simplement parce qu'ils étaient facilement accessibles. La perpétuation de cet état de choses retardera le progrès à la fois parce que l'exactitude des prédictions est tributaire de la pertinence et de la précision des mesures utilisées et parce que les chercheurs continueront d'utiliser les mêmes mesures quoiqu'ils les analyseront avec des techniques plus sophistiquées. Il semble donc logique d'affirmer que les mesures prédictives doivent être reliées à une certaine théorie. À son tour, toute théorie qui tente de prédire un comportement doit inclure des comptes rendus au sujet du ou des individus pouvant présenter le comportement à l'étude et les stimuli pouvant l'influencer. Le genre de réactions qu'ils démontrent à des stimuli variés est peut-être ce qui différencie le mieux les types d'individus. Cependant, afin de rendre cette approche situation-personne crédible, on doit démontrer, premièrement, qu'il existe des déterminants situationnels de la violence ainsi qu'une méthodologie d'enquête pour les étudier, deuxièmement, qu'il y a des différences individuelles dans la propension à commettre des actes de violence et, troisièmement, qu'une méthodologie existe pour examiner l'interaction entre ces deux variables.

\section{DIFFERENCES INDIVIDUELLES}

Dans le but de démontrer de façon convaincante les différences individuelles dans la propension à l'agressivité, il faut noter la fréquence des actes de violence pour un certain nombre d'individus dans une même situation, pendant une période de temps prolongée. Quinsey et Varney (1977) ont enregistré les agressions physiques survenues dans une unité psychiatrique à sécurité maximum de 150 lits. Ces enregistrements de comportement agressif étaient suffisamment complets et précis pour permettre d'observer les changements dans la fréquence des agressions chez un même patient, sur une période d'un an. On a constaté que $13 \%$ $(\mathrm{N}=18)$ des patients avaient commis $61 \%$ des 198 délits de violence et 
que la grande majorité des patients n'en avait commis aucun. Les quelques patients très agressifs étaient différents sous un certain nombre de rapports des patients non agressifs et, plus important encore, la fréquence élevée des actes de violence commis par eux s'est maintenue pendant de longues périodes. Douze des 18 patients avaient été très agressifs au cours de l'année qui a précédé l'étude et au moins quatre l'avaient déjà été l'année précédente. Le même genre de situations sociales, les taquineries et ce qui était perçu comme des provocations du personnel, a semblé avoir entraîné la plupart des actes de violence chez tous les patients très agressifs. Toch (1969) a obtenu des résultats semblables; il a constaté que les policiers, les gardiens de prison et les délinquants agressifs le demeuraient pour de longues périodes et réagissaient agressivement à des situations sociales spécifiques comme par exemple des menaces perçues à leur «réputation» ou à leur autorité. Ces données indiquent donc qu'il existe des patterns d'agressivité stables, propres à certains individus, qui sont reliés à certaines conditions du milieu de vie. 11 y a aussi de nombreuses preuves tirées d'études longitudinales que l'agressivité physique est un trait durable et stable (Farrington, 1982).

On peut évidemment formuler l'objection que ces études menées dans un milieu naturel n'ont pas contrôlé la possibilité que les variations dans la fréquence des agressions d'un individu à l'autre soient causées par des variations dans la fréquence des incidents provocateurs que les individus rencontraient. Il y a, cependant, au moins quelques indices à l'effet que les personnes très agressives réagissent agressivement à des stimuli qui ne provoquent pas de telles réactions chez d'autres individus. Ces indices seront examinés dans la section suivante.

\section{CONTRÔLE DES STIMULI}

Depuis longtemps, les criminologues savent que les crimes graves contre la personne ne se produisent pas au gré du hasard. Wolfgang (1958), dans son étude classique de l'homicide, a démontré que les meurtres ont plus de chances d'être commis par des hommes de race noire, âgés de 20 à 24 ans, le samedi soir entre $20 \mathrm{~h}$ et $2 \mathrm{~h}$, sur une voie publique. Les victimes avaient tendance à être de la même race que leur meurtrier, quoique plus âgées. De la même façon, Quinsey et Varney (1977) ont démontré que la probabilité qu'un crime de violence soit commis variait grandement selon les heures de la journée. Cependant, ces constatations n'indiquent que d'une façon très sommaire le contrôle de la violence par les stimuli.

Pour obtenir des preuves convaincantes du contrôle du stimulus, il faut procéder à un examen détaillé du contrôle du stimulus chez des 
individus. Malheureusement, aucune démonstration solide n'a été faite à propos des comportements agressifs graves; il y en a pourtant à propos des agressions moins graves. De telles données sont fournies par Patterson et Cobb (1973) qui ont, à diverses périodes, recueilli des informations quant à 29 catégories de comportements auprès de 26 familles sans problème et de 32 familles avec des garçons agressifs. On a constaté que tous les stimuli facilitant l'agressivité sociale étaient émis par les frères et soeurs; les parents freinaient cette forme de réaction ou n'avaient aucune influence sur elle. Les chercheurs ont conclu que : "ces procédés pouvaient identifier les stimuli sociaux qui contrôlaient certaines réactions sociales malsaines présentées par les garçons. Pour les garçons de familles à problèmes, la quantité minime de contrôle des stimuli significatifs témoigne de l'efficacité de cette approche. Les conclusions soulignent le fait que beaucoup (pas tous) de déterminants importants du comportement sont présents dans les stimuli sociaux immédiatement observables au cours des interactions sociales".

Dans une autre étude sur les enchaînements d'interactions sociales, Raush (1965) a déduit que les enchaînements d'interactions différenciaient les garçons normaux des hyperagressifs. Tout comme Patterson et Cobb, il a découvert que la réaction exprimée par un garçon affectait de façon importante la réaction de l'autre, et ceci dans les deux groupes, mais que les garçons hyperagressifs réagissaient à des propos amicaux par des paroles désobligeantes beaucoup plus fréquemment que les garçons normaux. Les deux groupes de garçons réagissaient à des remarques désobligeantes par des expressions hostiles. Bien que la faible dimension des échantillons et les différences entre les groupes maintiennent ces découvertes au stadè expérimental, elles suggèrent qu'il existe des différences individuelles qui se reflètent dans le type de réaction provoquée par un stimulus social spécifique.

\section{INTERACTION INDIVIDU-SITUATION}

Nous avons présenté quelques témoignages à l'effet qu'il existe des différences individuelles au niveau de l'agressivité, du contrôle des stimuli de l'agressivité et de l'interaction entre ces variables. Nous nous proposons maintenant d'étudier la façon dont ces interactions peuvent être analysées plus directement. Dans la section précédente, nous avons vu comment des typologies d'individus violents ont été élaborées à partir d'informations sur leurs délits, données qui permettaient de classer ces individus dans des catégories. Une autre façon de développer une taxonomie des individus violents serait d'observer leurs réactions face à différents types de stimuli; en d'autres mots, les individus seraient classés 
selon leurs réactions à certaines situations. Évidemment, le même problème de classification se pose pour les situations. Il est nécessaire que les situations soient classées en fonction du degré de similitude des comportements qu'elles provoquent. Essentiellement, nous suggérons que la théorie soit construite comme une matrice à trois dimensions qui comporterait les dimensions suivantes : individus, réactions, situations.

\section{UNE STRATÉGIE DE RECHERCHE}

À long terme, notre aptitude à prédire le comportement violent reposera sur des théories de l'agression qui s'appliquent à des personnes qui n'ont pas été internées, aussi bien qu'à des individus qui ont déjà été incarcérés pour des délits de violence. Une approche longitudinale semblerait offrir les meilleures perspectives pour comprendre comment les variables psychologiques et les variables sociales se conjuguent avec les variables situationnelles dans le développement d'un processus qui produit le comportement violent. Farrington (1982) a résumé quelques-uns des travaux des plus intéressants qui ont été menés dans ce domaine. L'étude de Wolfgang (1975) est un exemple de recherche longitudinale; il a suivi l'histoire de 9946 garçons nés en 1945 et élevés à Philadelphie. $30 \%$ des Blancs et $50 \%$ des Non-Blancs avaient eu au moins un contact avec la police avant l'âge de 18 ans. Seulement $6 \%$ des 9946 garçons avaient commis cinq délits ou plus, mais ces délinquants chroniques étaient responsables de $52 \%$ de tous les délits commis par cette cohorte. Plus important encore, ces délinquants chroniques étaient responsables de $53 \%$ des 815 délits de violence commis par tout le groupe. Enfin, on s'est aperçu qu'il y avait peu de spécialisation délictuelle à l'intérieur de tout le groupe. Les informations sur le délit ont aussi indiqué qu'un programme d'intervention serait inutile s`il s'adressait aux délinquants qui n'ont commis qu'un ou deux délits parce qu'une forte proportion d'entre eux ne récidive pas. Toutefois, il serait économiquement avantageux de concentrer ces efforts sur les délinquants à leur troisième délit à cause de la forte probabilité de récidive. L'étude de Wolfgang démontre que la recherche longitudinale est profitable du fait qu'elle révèle des informations à la fois pertinentes du point de vue théorique et ayant des implications sur les politiques sociales.

La recherche future dans ce domaine pourrait comporter la sélection de sous-groupes restreints, théoriquement homogènes, de délinquants chroniques et de non-délinquants pour des études spéciales. Dans ces études, des variables situationnelles telles les conditions de renforcement fournies par la famille et les pairs, les variables physiologiques et psychologiques et des données au sujet de la perception que le délinquant a de 
ses délits pourraient être intégrées dans un modèle de prédiction multivarié. Les variables identifiées dans ces secteurs fondamentaux de la recherche scientifique pourraient alors être utilisées pour des études de prédiction de sous-groupes particuliers d'individus en institution. Ce genre de recherche est multidisciplinaire, exige beaucoup de temps puisqu'elle est longitudinale et s'avère onéreuse; mais, si on se fie aux modestes progrès réalisés à ce jour, il semble que de nouveaux progrès seraient proportionnels à l'effort et aux fonds qui y seront investis.

\section{BIBLIOGRAPHIE}

BLACKBURN, R. (1968), "Personality in Relation to Extreme Aggression in Psychiatric Offenders", British Journal of Psychiatry, vol. 114, p. 821-828.

BOWERS, K. (1973), "Situations in Psychology : An Analysis and a Critique», Psycholo. gical Review, vol. 80, p. 307-336.

CAMPBELL, D.T. and D.W. FISHER (1959), "Convergent and Discriminant Validation by the Multitrait Multimethod Matrix", Psychological Bulletin, vol. 56, p. 81-105.

CARLSON, K.A. (1973), "Some Characteristics of Recidivists in an Ontario Institution for Adult Male First-Incarcerates", Canadian Journal of Criminology and Corrections, vol. 15 , p. 397-411.

CONRAD, J.P. (1966), «The Nature and Treatment of the Violent Offender: A Typology of Violence", Appendix 2 of C. Spencer, A Typology of Violent Offenders, California Department of Corrections, Research Report 23.

ENDLER, N.S. (1975), "The Case for Person-Situation Interactions", Canadian Psychological Review, vol. 16, p. 12-21.

FARRINGTON, D.P. (1982), "Longitudinal Analyses of Criminal Violence", in M.E. Wolfgang and N.A. Weiner (édit.), Criminal Violence, Beverly Hills, Sage.

FLYNN, J.P. (1967), "The Neural Basis of Aggression in Cats", in D.C. Glass (édit.), Neurophysiology and Emotion, New York, Rockfeller University Press.

FREDERIKSON, N. (1972), "Toward a Taxonomy of Situations", American Psychologist, vol. 27, p. 114-123.

GATHERCOLE, C.E., M.J. CRAFT, J. McDOUGALL, H.M. BARNES, D.F. PECK (1968) "A Review of 100 Discharges from a Special Hospital", British Journal of Criminology, vol. 8, p. 419-424.

GIBBENS, T.C.N., D.A. POND, D. STAFFORD -CLARK (1955), "A Follow-up Study of Criminal Psychopaths", British Journal of Delinquency, vol. 6, p. 126-135.

GIBBENS, T.C.N., D.A. POND, D. STAFFORD-CLARK (1959), «A Follow-up Study of Criminal Psychopaths", Journal of Mental Science, vol. 105, p. 108-115.

GLASER, D., D. KENEFICK, V. O'LEARY (1966), The Violent Offender, National Parole Institutes, Washington, U.S. Government Printing Office.

GOLDSTEIN, M. (1974), «Brain Research and Violent Behavior : A Summary and Evaluation of the Status of Biomedical Research on Brain and Aggressive Violent Behavior", Archives of Neurology, vol, 30, p. 1-35.

GOTTFREDSON, D.M., L.T. WILKINS, P.B. HOFFMAN (1978), Guidelines for Parole and Sentencing : A Policy Control Method, Toronto, Lexington Books. 
GREENWOOD, P.W. and A. ABRAHAMSE (1982), Selective Incapacitation, Santa Monica, Rand.

GULEVICH, G.D. and P.G. BOURNE (1970), "Mental Illness and Violence», in D.N. Daniels, M.F. Gilula and F.M. Ochburg (édit.), Violence and the Struggle for Existence, Boston, Little Brown.

HEDLUND, J.L., I.W. SLETTEN, H. ALTMAN, R.C. EVENSON (1973), «Prediction of Patients Who Are Dangerous to Others", Journal of Clinical Psychology, vol. 29, p. 443.447.

HENSERSON, M. (1983), "Self-Reported Assertion and Aggression Among Violent Offenders with High or Low Levels of Overcontrolled Hostility ", Personality and Individual Differences, vol. 4, p. 113-115.

HOLLAND, T.R., N. HOLT, M. LEVI, G.E. BECKETT (1983), "Comparison and Combination of Clinical and Statistical Predictions of Recidivism Among Adult Offenders", Journal of Applied Psychology, vol. 68, p. 203-211.

JOHNSON, J.H. (1974), "A Cross-Validation of Seventeen Experimental MMPI Scales Related to Antisocial Behavior», Journal of Clinical Psychology, vol. 30 , p. 564-565.

KAHNEMAN, D. and A. TVERSKY (1973), "On the Psychology of Prediction", Psychological Review, vol. 80, p. 237-251.

KOZOL, H.L. (1975), "The Diagnosis of Dangerousness", in S.A. Pasternack (édit.), Violence and victims, New York, Spectrum.

KOZOL, H.L., R.J. BOUCHER, K.F. GARAFALO (1972), "The Diagnosis and Treatment of Dangerousness", Crime and Delinquency, vol. 18, p. 371-392.

LLOYD, C.W. and J. WEISZ (1975), "Hormones and Aggression", in W.S. Fields and W.H. Sweet (édit.), Neural Bases of Violence and Aggression, St. Louis, Mo., Green.

McGARRY, A.L. (1971), "The Fate of Psychotic Offenders Returned for Trial», American Journal of Psychiatry, vol. 126, p. 1181-1184.

MEDNICK, S.A., V. POLLOCK, J. VOLAVKA, W.F. GABRIELLI (1982), "Biology and Violence", in M.E. Wolfgang and N.A. Weiner (édit), Criminal violence, Beverly Hills, Sage.

MEGARGEE, E.I. (1969), «A Critical Review of Theories of Violence», in D.J. Mulvihill, M.M. Tumin and L.A. Curtis (édit.), Crimes of Violence, vol. 13, U.S. Government Printing Office.

MEGARGEE, E.I. (1970), "The Prediction of Violence With Psychological Tests", in C.D. Spielberger (édit), Current Topics in Clinical and Community Psychology, vol. 2, New York, Academic Press.

MEGARGEE, E.I. (1982), «Psychological Determinants and Correlates of Criminal Violence", in M.E. Wolfgang and N.A. Weiner (édit.), Criminal Violence, Beverly Hills, Sage, 1983.

MEGARGEE, E.I., P.E. COOK, G.A. MENDELSOHN (1967), "Development and Evaluation of an MMPI Scale of Assaultiveness in Overcontrolled Individuals", Journal of Abnormal Psychology, vol. 72, p. 519-528.

MESNIKOFF, A. and C.G. LAUTERBACH (1975), "The Association of Violent Dangerous Behavior With Psychiatric Disorders : A Review of the Research Literature", Journal of Psychiatry and Law, vol. 31, p. 415-445.

MOLOF, M.J. (1965), Prediction of Future Assaultive Behavior Among Youthful Offenders, California Department of Youth Authority Research, Report 41. 
MONAHAN, J. (1978), "Prediction Research and the Emergency Commitment of Dangerous Mentally Ill Persons : A Reconsideration", American Journal of Psychiatry. vol. 135, p. 199-202.

MONAHAN, J. (1981), Predicting Violent Behavior : An Assessment of Clinical Techniques, Beverly Hills, Sage.

MONAHAN, J. and D. KLASSER (1982), "Situational Approaches to Understanding and Predicting Individual Violent Behavior", in M.E. Wolfgang and N.A. Weiner (ëdit.), Criminal Violence, Beverly Hills, Sage.

MORRISON, W.A. (1973), "Criminal Homicide and the Death Penalty in Canada : Time for Re-Assessment and New Directions : Toward a Typology of Homicide", Canadian Journal of Criminology and Corrections, vol. 15, p. 367-396.

MORROW, W.R. and D.B. PETERSON (1966), "Follow-up of Discharged Psychiatric Offenders - "Not Guilty by Reason of Insanity" and "Criminal Sexual Psychopaths", Journal of Criminal Law, Criminology and Police Science, vol. 57 , p. 31-34.

NUFFIELD, J. (1982), Parole Decision-Making in Canada, Ottawa, Ministry of Supplies and Services.

PATTERSON, G.R. and J.A. COBB (1973), "Stimulus Control for Classes of Noxious Behaviors", in J.F. Knutson (édit.), The Control of Aggression. New York, Aldine.

PRUESSE, M.G. and V.L. QUINSEY (1977), "The Dangerousness of Patients Released from Maximum Security : A Replication ", Journal of Psychiatry and Law, vol. 5, p. 293-299.

QUINSEY, V.L. (1977), "The Assessment and Treatment of Child Molesters : A Review", Canadian Psychological Review, in press.

QUINSEY. V.L. (1979a), «Demographic and Clinical Variables Associated With Release From a Maximum Security Psychiatric Institution “, Criminal Justice and Behavior, vol. 6 , p. $390-399$.

QUINSEY, V.L. (1979b), "A Review of "The Criminal Insane", by T. Thomberry and J. Jacoby, Journal of Psychiatry and Law, vol. 7, p. 279-283.

QUINSEY, V.L. (1980), «The Baserate Problem and the Prediction of Dangerousness : A Reappraisal", Journal of Psychiatry and Law, vol. 8, p. 329-340.

QUINSEY, V.L. (1983), "Prediction of Recidivism and the Evaluation of Treatment Programs for Sex Offenders", in S. Simon-Jones and A.A. Keltner (édit.), Sexual Aggression and the Law, Criminology Research Centre, Simon Fraser University.

QUINSEY, V.L. (in press), «Sexual Aggression : Studies of Offenders Against Women», International Yearbook on Law and Mental Health.

QUINSEY, V.L., R. AMBTMAN, M. PRUESSE (1977), «Institutional Release Policy and the Identification of Dangerous Men», Presented at the Symposium on Violence in Canadian Society, Qualicum Beach, Vancouver Island.

QUINSEY, V.L., R. AMBTMAN (1978), «Psychiatric Assessments of the Dangerousness of Mentally Ill Offenders», Crime and Justice, vol. 6, p. 249-257.

QUINSEY, V.L., R. AMBTMAN (1979), "Variables Affecting Psychiatrists` and Teachers' Assessments of the Dangerousness of Mentally Ill Offenders", Journal of Consulting and Clinical Psychology, vol. 47, p. 353-362.

QUINSEY, V.L. and T.C. CHAPLIN (1982), "Penile Responses to Nonsexual Violence Among Rapists", Criminal Justice and Behavior, vol. 9, p. 312-324.

QUINSEY, V.L., A MAGUIRE, G.W. VARNEY (1983), "Assertion and Overcontrolled Hostility Among Mentally Disordered Murderers", Journal of Consulting and Clinical Psychology, vol. 51, p. 550-556. 
QUINSEY, V.L., M. PRUESSE, R. FERNLEY (1975a), «A Follow-up of Patients Found not Guilty Because of Insanity or Unfit for Trial", Canadian Psychiatric Association Journal, vol. 20, p. 461-467.

QUINSEY, V.L., M. PRUESSE, R. FERNLEY (1975b), "Oak Ridge Patients : Prerelease Characteristics and Postrelease Adjustment *, Journal of Psychiatry and Law, vol. 3, p. 63-73.

QUINSEY, V.L., C.S. STEINMAN, S.G. BERGERSEN, T.F. HOLMES (1975), „Penile Circumference, Skin Conductance and Ranking Responses of Child Molesters and "Normals" to Sexual and Non-sexual Visual Stimuli", Behavior Therapy, vol. 6, p. $213 \cdot 219$.

QUINSEY, V.L. and G.W. VERNEY (1977), "Characteristics of Assaults and Assaulters in a Maximum Security Psychiatric Unit", Crime and Justice, vol. 5, p. 212-220.

QUINSEY, V.L., A. WARNEFORD, M. PRUESSE, N. LINK (1975), « Released Oak Ridge Patients : A Follow-up of Review Board Discharges", British Journal of Criminology, vol. 15, p. 264-270.

RAPPEPORT, J.R. (ed.) 1967), The Clinical Evaluation of the Dangerousness of the Mentally $\mathrm{Ill}$. Springfield, Illinois, Thomas.

RAUSH, H.L. (1965), "Interaction Sequences", Journal of Personality and Social Psychology, vol. 2, p. 487-499.

RAZAVI, L. (1975), "Cytogenic and Somatic Variation in the Neurobiology of Violence : Epidemiological, Clinical and Morphogenetic Considerations", in S.W. Fields and W.H. Sweet (édit.), Neural Bases of Violence and Aggression, St. Louis, Mo., Green.

RUBIN, B. (1972), "Prediction of Dangerousness in Mentally lll Criminals", Archives of General Psychiatry, vol. 27, p. 397-407.

SHAH, S.A. (1975), "Dangerousness and Civil Commitment of the Mentally 111 : Some Public Policy Considerations", American Journal of Psychiatry, vol. 132, p. 501-505.

SPENCER, C. (1966), A Typology of Violent Offenders, California Department of Corrections, Research Report 23.

STEADMAN, H.J. (1976), "Predicting Dangerousness", in D.J. Madden and J.R. Lion (édit.), Rage, Hate, Assault, and Other Forms of Violence, Spectrum.

STEADMAN, H.J. (1982), "A Situational Approach to Violence", International Journal of Law and Psychiatry, vol. 5, p. 171-186.

STEADMAN, H.J. and J.J. COCOZZA (1974), Careers of the Criminally Insane, Lexington, Mass., Lexington Books.

THORNBERRY, J.P. and J.E. JACOBY (1979), The Criminally Insane : A Community Follow-up of Mentally $I l l$ Offenders, Chicago, University of Chicago Press.

TOCH, H. (1969), Violent Men : An Inquiry Into the Psychology of Violence, Chicago, Aldine.

TONG, J.E. and J.W. McKAY (1959), "A Statistical Follow-up of Mental Defectives of Dangerous or Violent Propensities" British Journal of Delinquency, vol. 9, p. 276-284.

TUCKER, L.R. (1966), "Some Mathematical Notes on Three-Mode Factor Analysis", Psychometrika, vol. 31, p. 279-311.

WALKER, N., W. HAMMOND. D. STEER (1967), "Repeated Violence, The Criminal Law Review, vol. 207, p. 465-472.

WALKER, N.D. and S.F. McCABE (1973), Crime and Insanity in England, vol. 2, Edinburgh University Press. 
WALLER, I. (1974), Men Released From Prison, University of Toronto Press.

WALTERS, G.D. and R.L. GREENE (1983), "Factor Structure of the OvercontrolledHostility Scale of the MMPI", Journal of Clinical Psychology, vol. 39, p. 560-562.

WEBSTER, C. and B. DICKENS (1983), Deciding Dangerousness : Policy Alternatives for Dangerous Offenders, Department of Justice.

WENK, E.A. and R.L. EMRICH (1972, Assaultive Youth : An Exploratory Study of the Assaultive Experience and Assaultive potential of California Youth Authority Wards, U.S. National Institute of Law Enforcement and Criminal Justice.

WENK, E.A., J.O. ROBISON, G.W. SMITH (1972), «Can Violence Be Predicted?», Crime and Delinquency, vol. 18, p. 393-402.

WEXLER, D.B. (1976), Criminal Commitment and Dangerous Mental Patients : Legal Issues of Confinement, Treatment, and Release, National Institute of Mental Health, DHEW Publication $\mathbf{n}^{\text {" }}$ (ADM) 77-331, sup. of Documents, U.S. Govemment Printing Office.

WHITE, W.C., W.G. McADOO, E.I. MEGARGEE (1973), "Personality Factors Associated With Over and Undercontrolled Offenders», Journal of Personality Assessment, vol. 37, p. 473-478.

WILKINS, L.T. (1973), Ineffecient Statistics, Supplemental Report Six, Davis, California, Parole-Decision-Making Project, National Council on Crime and Delinquency Center, Davis, California.

WOLFGANG, M.E. (1975), «Delinquency and Violence From the Viewpoint of Criminology", in W.S. Fields and W.H. Sweet (édit.) Neural Bases of Violence and Aggression, St. Louis, Mo., Green.

WOLFGANG, M.E. (1958), Patterns in Criminal Homicide in Philadelphia, University of Pennsylvania. 